\title{
miR-146a-5p mediates epithelial- mesenchymal transition of oesophageal squamous cell carcinoma via targeting Notch2
}

Cong Wang, Wenxue Zhang, Lin Zhang, Xuan Chen, Fang Liu, Jing Zhang, Shanghui Guan, Yi Sun, Pengxiang Chen, Ding Wang, Effat Un Nesa, Yufeng Cheng and George M Yousef

Correction to: British Journal of Cancer (2016) 115, 1548-1554. doi:10.1038/bjc.2016.367; published online 10 November 2016

Since publication of this manuscript, it was recognised that there is an error in Figure 3D and $\mathrm{E}$ of the results. The correct figure is presented here.

The sample labels for 'anti-Notch 2' and 'NC' were incorrectly placed for the EC 9706 and Eca 109 cell lines in Figure 3D and E, and the protein 'vimentin' was incorrectly labelled as 'N-Cadherin' in Figure 3D. The results are correctly described in the main text of the manuscript, and the incorrect figure had no effect on the overall study conclusions.

This work is published under the standard license to publish agreement. After 12 months the work will become freely available and the license terms will switch to a Creative Commons AttributionNonCommercial-Share Alike 4.0 Unported License. 
A

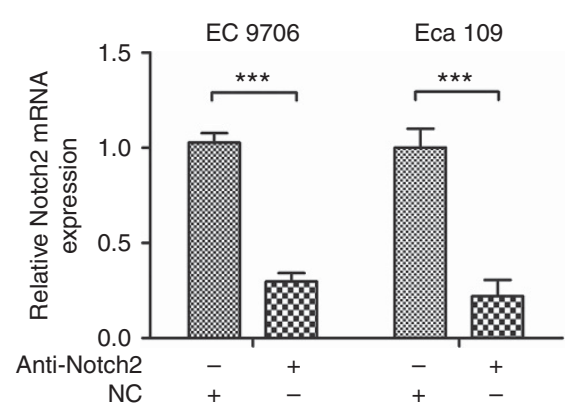

C

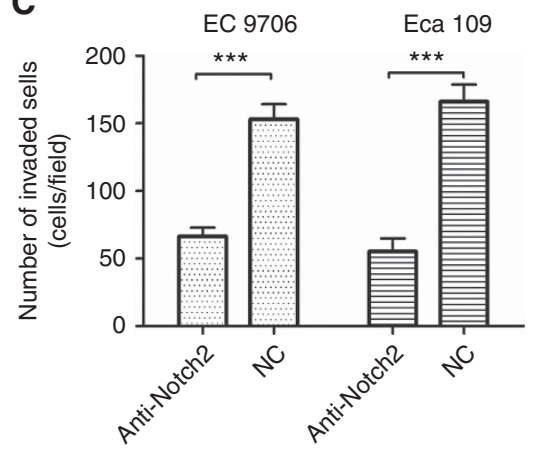

E

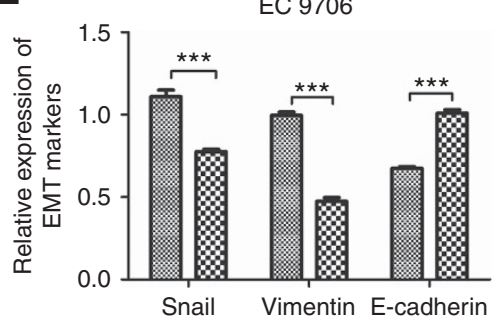

B

EC 9706
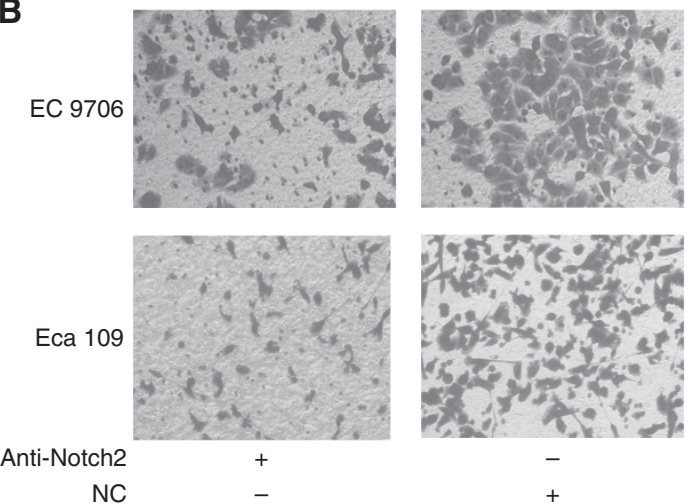

$+$

D

$$
\text { Snail }
$$

EC 9706

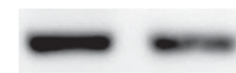

Vimentin
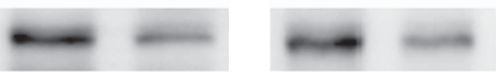

E-cad
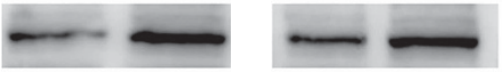

$\beta$-actin

Anti-Notch2

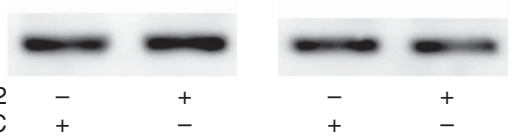

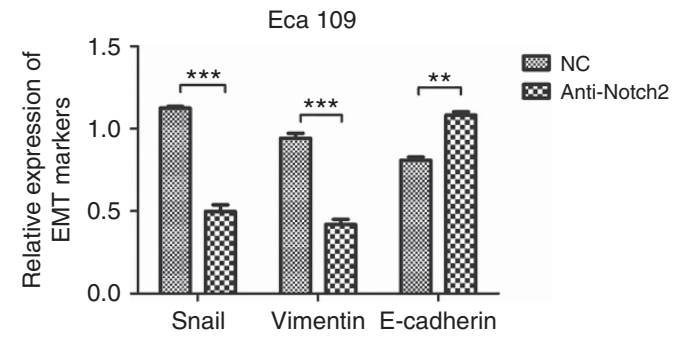

Figure 3. Notch2 promotes EMT of ESCC. (A) shRNA transfection of Notch2 could inhibit its expression. (B, C) shRNA-Notch2 inhibited invasion ability of ESCC. (D, E) shRNA-Notch2 regulated EMT markers of ESCC. ${ }^{\star *} P<0.01$; ${ }^{\star \star *} P<0.001$. 